OPEN

SUBJECT AREAS:

MOLECULAR

ENGINEERING

GENE THERAPY

VIROLOGY

TUMOUR SUPPRESSORS

Received

30 November 2011

Accepted

12 March 2012

Published

11 April 2012

Correspondence and requests for materials should be addressed to J.K. (ajkomano@nih. go.jp; komano@iph. pref.osaka.jp)

\footnotetext{
* Current address:

Research Center for

Allergy and

Immunology, RIKEN

Yokohama Institute,

1-7-22 Suehiro-cho,

Tsurumi, Yokohama,

Kanagawa, 230 -

0045, Japan.
}

"Current address:

Osaka Prefectural

Institute of Public

Health, Dept. Of

Infectious Diseases,

3-69, Nakamachi,

1-chome Higashinari-

ku, Osaka, 537-0025,

Japan.

\section{Therapeutic potential of HIV protease-activable CASP3}

\author{
Kosuke Miyauchi*, Emiko Urano, Mari Takizawa, Reiko Ichikawa \& Jun Komano\#
}

AIDS Research Center, National Institute of Infectious Diseases, 1-23-1 Toyama, Shinjuku-ku, Tokyo 162-8640, Japan.

Development of a therapeutic application of CASP3/caspase 3/CPP32, an executor of apoptosis, has been challenging because regulation of its activation is complicated. This study aimed to inhibit cancer cell growth and human immunodeficiency virus type 1 (HIV-1) propagation through a CASP3 mutant, CASP3*, activable by HIV-1-encoded aspartate protease. Active CASP3* was delivered to leukemic cells using a protein transduction vehicle, the lentivirus-like nanoparticle (LENA), which should contain thousands of CASP3*-Gag protein molecules and release the activated CASP3* into the target cell cytoplasm. CASP3*-LENA induced apoptosis in various types of leukemic cells. In addition to being effective against leukemic cells, constitutive expression of CASP3* restricted HIV-1 propagation in SUP-T1 cells. The attenuation of HIV-1 replication in SUP-T1/CASP3* cells was attributed to the elimination of HIV-1-infected cells by apoptosis. These data suggest that CASP3* has therapeutic potential against both lymphoid malignancies and HIV-1 infection.

ASP3 is expressed as an inactive pro-enzyme that is activated upon exposure to apoptosis-inducing stimuli ${ }^{1,2}$. Pro-CASP 3 undergoes proteolytic processing by CASP 8,9 and 10 that yields three polypeptides: the pro domain, $\mathrm{p} 17$ and $\mathrm{p} 12$. The $\mathrm{p} 17$ and $\mathrm{p} 12$ form a heterodimer that executes the protease activity. CASP3 activates itself as well as CASP6, 7 and 9 by proteolytic cleavage and amplification of the signal for the execution of apoptosis. The therapeutic application of CASP3 is limited because of this complex regulation ${ }^{3-5}$. We overcome this problem by genetic engineering the CASP3.

Here, a mutant of CASP3 designed to be activated specifically by the aspartate protease of human immunodeficiency virus type 1 (HIV-1), but not by other CASPs, was produced (CASP $3 *$ ) and a proof-of-concept study was conducted to demonstrate the therapeutic potential of CASP3* against lymphoid malignancies and HIV-1 infection.

To achieve leukemic cell killing by CASP3*, a lentivirus-like nanoparticle (LENA) system was utilized ${ }^{6}$. The LENA system is a simple, efficient and reproducible method that we have developed to transduce proteins into mammalian cells ${ }^{6}$. The LENA is different from lentiviral vector in that the former system delivers proteins that are encapsidated into the nanoparticles but not genes as does the latter. Protein transduction does not require de novo transcription and translation, and the transferred protein functions immediately after the transduction. Also, LENA is biologically safe since LENA is not an "infectious" agent. Approximately 5,000 CASP 3 *-Gag proteins are packaged, processed and activated by HIV-1 protease in the particle of LENA. CASP3*-LENA, facilitated by vesicular stomatitis virus G protein (VSV-G), binds to cells and enters them via endocytosis. Membrane fusion between the cell and LENA takes place at the endosome in a VSV-G-dependent manner. The LENA content is then released into the cell cytoplasm. We expected an initiation of apoptosis in CASP3*-LENA-exposed leukemic cells immediately after membrane fusion.

In the HIV/AIDS field, clinical trials have proved that gene therapy approaches are indeed effective against HIV-1 infection ${ }^{7,8}$. However, the emergence of treatment-resistant viruses is problematic, since HIV-1 is a highly mutagenic virus. Also, the "off-target" effect of therapeutic molecules is a serious concern. Thus, developing a highly specific therapeutic gene against HIV-1 provides another option for treatment of HIV-1-infected individuals in a molecular therapy approach.

In this study, the genetically-engineered CASP3 activated specifically by HIV-1 protease was shown to have therapeutic potential against both lymphoid malignancies and HIV-1 infection.

\section{Results}

CASP3* has proteolytic cleavage sites for HIV-1 protease adopted from the matrix (MA or $\mathrm{p} 17^{\mathrm{MA}}$ )-capsid (CA or p24 $4^{\mathrm{CA}}$ ) junction of HIV-1 Pr55 $5^{\mathrm{Gag}}$ (Gag, Fig. 1a). The myristoylation signal of Lyn was attached at the amino-terminus and serves as a membrane-targeting signal. The pro domain of CASP3 was dispensable for enzyme activity and was removed from this construct. Then, the CASP3* was applied to the LENA system for the leukemic cell killing experiment (Fig. 1b). The CASP3*-Gag and its proteolytic products were detected in the 


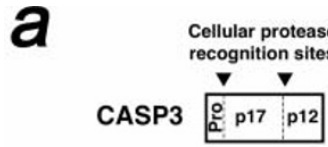

$b$

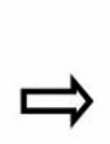

Myrlyn signal
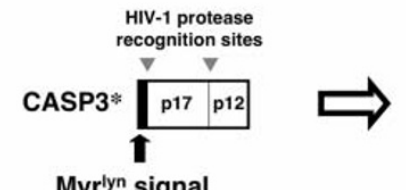

Active CASP3*
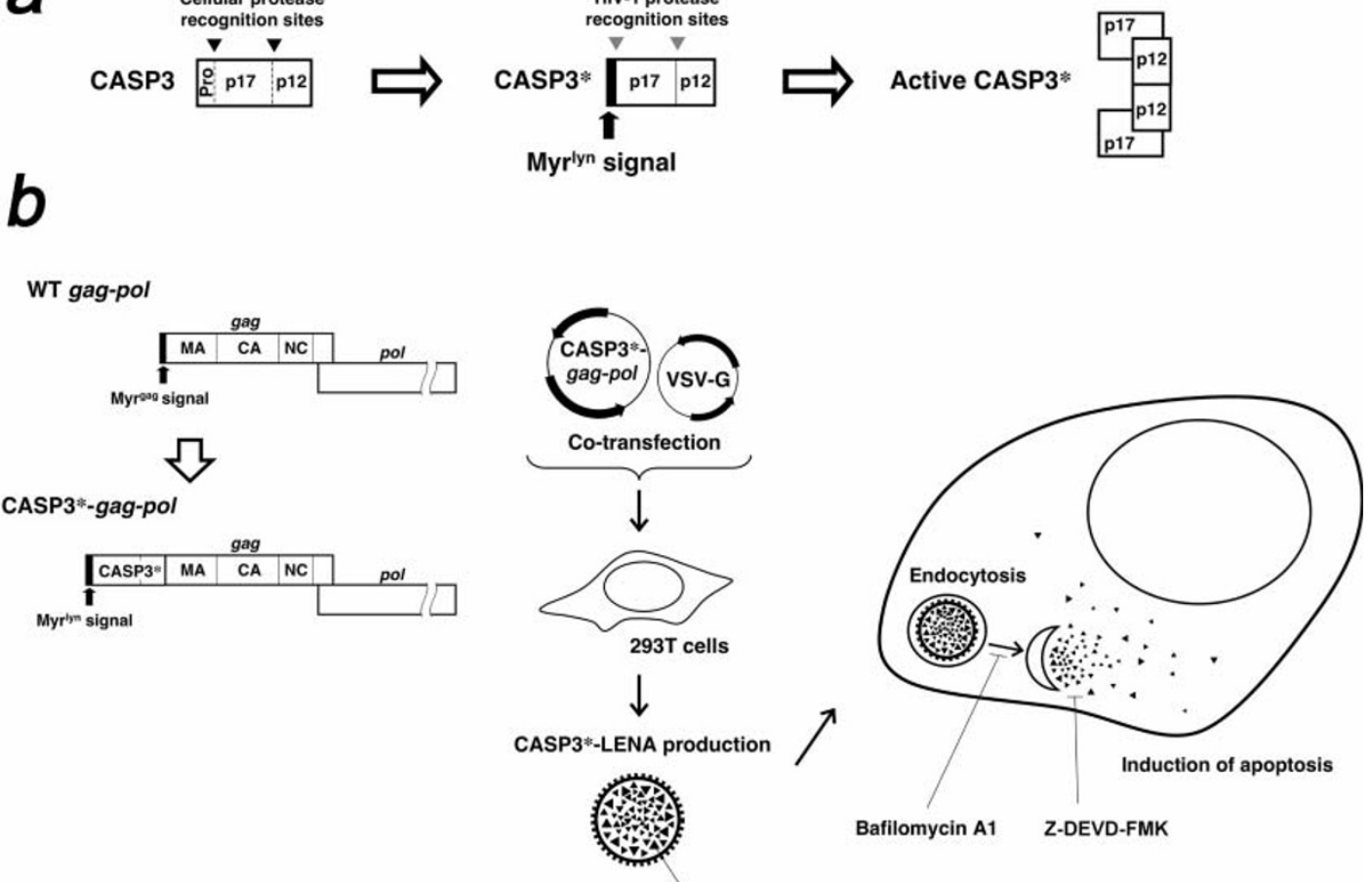

Activated CASP3*

C
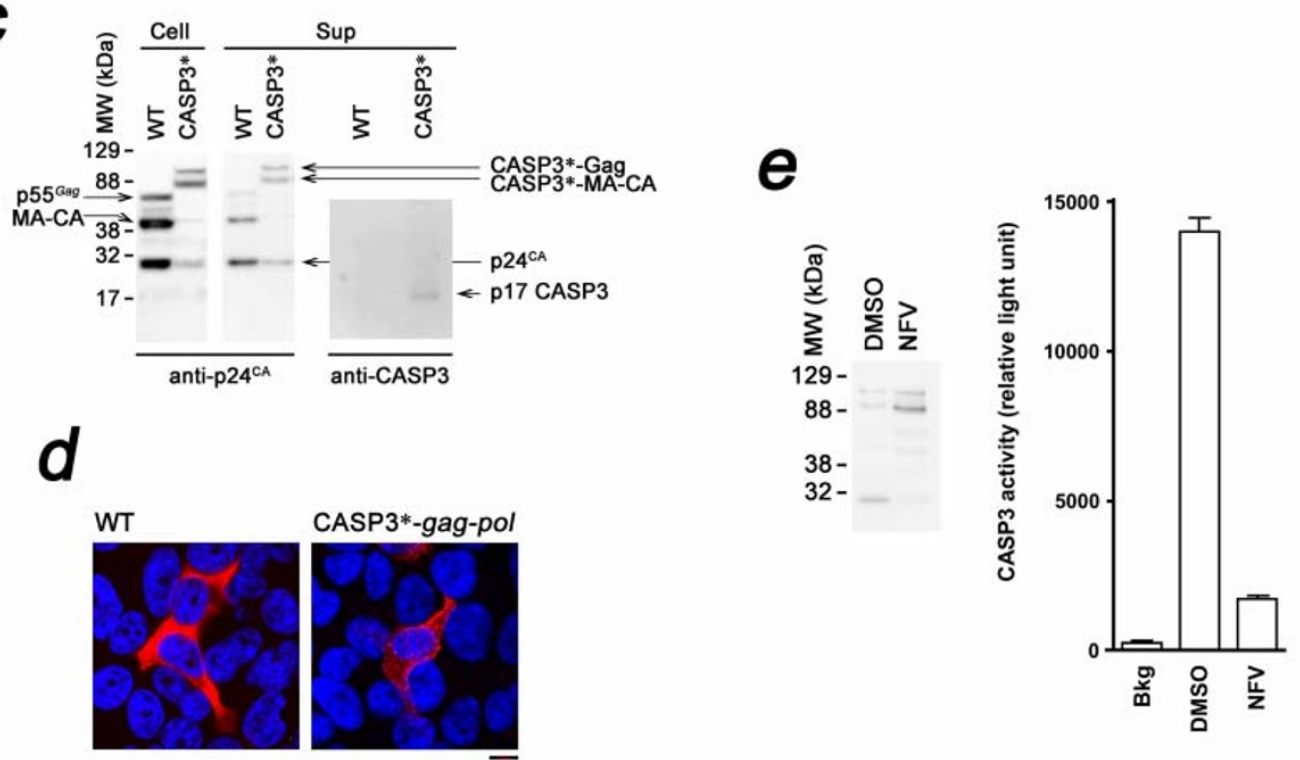

Figure 1 Construction, production and characterization of CASP3*-LENA. (a) Genetic structure of CASP3 and the genetic modification to produce HIV-1 protease-activable CASP3*. The cellular (gray triangle, digesting D28-S29 and D175-S176 junctions) and HIV-1 (black triangle) protease proteolytic sites are indicated. The CASP3 D28 was replaced with amino acids 127-136 (VSQNYPIVQ) from HIV-1 Gag (according to the HXB2 coordinate). The amino acids 129-134 (QNYPIV) from HIV-1 Gag according to the HXB2 coordinate was inserted after CASP3 D175. HIV-1 protease targets the YP junction. The enzyme is active when the p17 and p12 subunits are dimerized. (b) Production of CASP3*-LENA and its mechanism of action. The CASP3* was placed at the 5' end of wild type (WT) gag-pol to yield CASP3*-gag-pol. The proteolytic cleavage sites within Gag for HIV-1 protease and the proteolytic products are indicated MA, matrix; CA, capsid; and NC, nucleocapsid. The CASP3*-gag-pol and VSV-G expression vectors were co-transfected into 293 T cells. Then, the VSV-G-encapsidated LENA containing activated CASP3* protein was produced in the culture supernatant. CASP3*-LENA enters cells via an endocytotic route, with the content released from endosomes at the site of membrane fusion. The action points of Bafilomycin A1 (BAF) and the CASP3 inhibitor Z-DEVD-FMK are indicated. (c) Expression of CASP3*-gag-pol and CASP3*-LENA production. The cell lysates (Cell) and culture supernatants (Sup) of $293 \mathrm{~T}$ cells transfected with either wild type (WT) or CASP $3^{*}$-gag-pol (CASP3*) expression vector were analyzed by Western Blot. The corresponding protein for each band is indicated. (d) Immunofluorescence assay showing the distribution of WT Gag or CASP3*-Gag (CASP3*) in 293T cells transfected with either WT or CASP3*-gag-pol (CASP3*) expression vector. Red and blue represent the anti-p24 ${ }^{\text {CA }}$ monoclonal antibody-stained signal and the Hoechst 33258-stained nucleus, respectively. Magnification, x630; scale bar, $10 \mu \mathrm{m}$. (e) Detection of CASP3 enzyme activity in purified CASP3*-LENA. CASP3*-LENA was produced either in the presence or absence of nelfinavir (NFV) and collected by ultracentrifugation over a 20\% sucrose layer. DMSO was used as a control. The amount of CASP $3 *$-LENA and the cleavage pattern of Gag were examined by Western blot (left). CASP3 enzyme activity was detected in the CASP $3 *$-LENA lysate (right). 
293T cell lysate transfected with pCASP3*-gag-pol by Western blot analysis (Fig. 1c, Cell) in a pattern similar to that of wild-type Gagpol (WT, Fig. 1c). However the processing efficiency of Gag was slightly attenuated in the CASP3* construct compared with WT, as highlighted by the smaller amount of $\mathrm{p} 24^{\mathrm{CA}}$ relative to its precursor. In the culture supernatant of transfected 293T cells, CASP3*LENA was detected by Western blot analysis (Fig. 1c, Sup). The presence of CASP* was verified by Western blot analysis using anti-CASP3 antibody that specifically recognizes the p17 subunit of CASP3 (Fig. 1c, Sup). In 293T cells transiently transfected with the WT expression plasmid, Gag was evenly distributed in the cell cytoplasm as visualized by an immunofluorescence assay (Fig. 1d). In contrast, CASP3*-Gag was distributed mainly in the cytoplasm and, to a lesser extent, in the nucleus, forming numerous fine aggregations (Fig. 1d). Also, some CASP3*-Gag signal was detected at the cell periphery (Fig. 1d). Despite these differences, LENA production by CASP $3 *-$ Gag-pol was as efficient as that by WT; the signal ratio of CASP3*-Gag and its proteolytic products in the virus-like particle (VLP) fraction relative to the cell lysate were comparable to that of the WT (Fig.1c).

To verify the enzyme activity of CASP $3 *$ in LENA, the proteolytic activity of CASP3* was measured using a caspase 3/7-specific substrate, DEVD-aminoluciferin, that luminesces upon cleavage. Significant enzymatic activity was detected in the purified CASP3*-LENA (Fig. 1e). Importantly, this signal was substantially reduced when proteolytic cleavage of CASP ${ }^{*}$-Gag was attenuated through the preparation of CASP $3 *$-LENA in the presence of $0.5 \mu \mathrm{M}$ HIV-1 protease inhibitor nelfinavir (NFV; Fig. 1e). These data suggest that active CASP3* is enveloped in the LENA and that the activation of CASP $3 *$ is dependent on HIV-1 protease.

HIV-1 protease is fully activated after viral budding. Thus, the majority of CASP $3 *$ should be activated outside cells. This minimizes damage to LENA-producing cells by CASP3*. However, LENAproducing $293 \mathrm{~T}$ cells were damaged upon Gag-pol expression presumably due to the cytotoxicity of the small amount of HIV-1 protease activated in the cell cytoplasm upon overexpression of the protein. Cell viability and LENA production improved when the LENA-producing cells were treated with a low dose of HIV-1 protease inhibitor. Preparation of LENA in the presence of $0.2 \mu \mathrm{M}$ saquinavir (SQV) increased the LENA yield by approximately 4-fold (Fig. 2a). Under these conditions, HIV-1 protease in LENA remained active as evidenced by the production of cleavage intermediates of CASP3*-Gag (Fig. 2a). The activation of CASP3* was not blocked under these conditions because a cleavage intermediate, MA-CA, predicated the release of CASP $3 *$ from the precursor (Fig. 2a, arrowhead), and the biological activity of CASP3*-LENA was detected as described below. Similar data were obtained using the low dose of NFV.

For leukemic cell killing by CASP3*-LENA, an acute T cell lymphoblastic leukemia (ALL) cell line, MOLT-4, was chosen. MOLT-4 cell viability was significantly attenuated when cells were exposed to CASP3*-LENA, whereas no cell killing was observed in WT-LENA or non-enveloped CASP3*-LENA at 1d post-LENA exposure (Fig. $2 \mathrm{~b}$ and $2 \mathrm{c}$ ). A cell metabolism-based cell viability assay revealed that $93.3 \%$ of cell viability was lost in CASP3*-LENA-exposed MOLT-4 cells at 1d post-LENA exposure (Fig. 2b). This cell killing was more efficient when cells were treated with CASP3*-LENA prepared in the presence of $0.2 \mu \mathrm{M}$ SQV bearing a higher LENA titer (99.2\%, Fig. 2b). The MOLT-4 cells exposed to CASP3*-LENA contained fragmented nuclei with condensed chromatin, characteristic of cells undergoing apoptosis (Fig. 2d). Such nuclear morphology was not found in MOLT-4 cells exposed to WT-LENA or nonenveloped CASP3*-LENA. Induction of apoptotic cell death was detected at $6 \mathrm{~h}$ post-LENA exposure by Annexin $\mathrm{V}$ staining (Fig. 2e). The VSV-G functional inhibitor Bafilomycin A1, which prevents the endosome acidification required for the activation of
VSV-G, rescued the cells from apoptotic cell death at $12.5 \mathrm{ng} \mathrm{ml}^{-1}$ (Fig. 2c-f). A cell-permeable CASP3 inhibitor, Z-DEVD-FMK, also protected cells from CASP3*-LENA-induced apoptosis at $0.5 \mu \mathrm{M}$ (Fig. 2c-g). These data suggest that the cell death induced by CASP3*-LENA is dependent on VSV-G function and the proteolytic function of CASP $3 *$. The death inducing titer of CASP $3 *$-LENA for MOLT- 4 cell was $2.0 \times 10^{6}$ particles per milliliter, which was estimated from the dose-dependent cell killing kinetics using serially diluted CASP3*-LENA preparations (average of four independent experiments).

In addition to MOLT- 4 cells, CASP3*-LENA induced apoptosis in two more leukemic cell models, SUP-T1 cells, a pleural effusion T cell lymphoblastic lymphoma cell line, and the HTLV-1-transformed T cell line M8166, a model for adult T cell leukemia (ATL, Fig. 2g). Similar data were obtained in the human chronic myelogenous leukemia (CML)-derived cell line K562 and the neuroblastoma cell line NP-2 (data not shown). These data indicate that the cell killing activity of CASP3*-LENA is not restricted to the leukemic cells. In these experiments, WT-LENA and non-enveloped CASP3*-LENA failed to induce apoptosis. These data demonstrate the attractiveness of CASP3* protein transduction by LENA as a tool for molecular cancer therapy. The cell killing activity of CASP3*-LENA was not detected in primary blood mononuclear cells (data not shown). This is presumably because the primary cells are inefficient to the LENAmediated protein delivery and/or to the CASP3-induced apoptosis, or both. This suggests that the CASP3*-LENA may favor cancer cells to induce apoptotic cell death.

CASP3* was also applied to limit HIV-1 replication. We hypothesized that CASP3*-expressing $\mathrm{T}$ cells may be more susceptible to apoptosis upon HIV-1 infection due to the expression of HIV-1 protease in infected cells. To this end, SUP-T1 cells were transduced with CASP3* using a murine leukemia virus (MLV) vector, and two independently isolated pairs of SUP-T1 cells were analyzed (Fig. 3a). The rate of cell proliferation and the susceptibility to apoptosis induced by a serim depriviation in SUP-T1/CASP3* cells were indistinguishable from those in SUP-T1/Cont cells (data not shown). Cells were exposed to a low- or high-dose HIV-1 preparation and viral replication was monitored. In both isolates, HIV-1 propagation in SUP-T1/CASP3* cells was attenuated regardless of the HIV-1 exposure dose (Fig. 3b). These data suggest that CASP3* can limit the propagation of HIV-1 in tissue culture.

To determine the mechanism by which HIV-1 replication is blocked, the viral entry phase and the production phase were investigated separately. If the virus-associated protease is responsible for the activation of CASP3* at the viral entry phase, infection with the HIV-1-based lentiviral vector should also kill SUP-T1/CASP3* cells. Thus, the establishment efficiency of lentiviral vector infection in SUP-T1/CASP3* cells should be lower than that in control cells. To test this, SUP-T1/CASP3* cells were infected with a lentiviral vector expressing luciferase upon establishment of infection. The luciferase activity in SUP-T1/CASP3* cells at $4 \mathrm{~d}$ post-infection was lower than in the control cells (Fig. 3c). On the other hand, the levels of MLV-transduced luciferase activity in SUP-T1/ CASP3* cells were similar to those in the control cells (Fig. 3c). Note that CASP3* was not activated by MLV since the substrate preference of MLV protease is distinct from that of HIV-1 protease. These data suggest that virus-associated HIV-1 protease is capable of activating CASP3* to induce apoptosis in SUP-T1/CASP3* cells. Luciferase activity was still detected in the lentiviral vector-infected SUP-T1/CASP3* cells (Fig. 3c), implying that some cells might have lost the high-level expression of CASP $3 *$ or a single virion-associated protease might not be able to initiate CASP3* activation at levels sufficient to kill all of the infected cells. Next, the induction of apoptosis by the virion-associated viral protease was examined directly. SUP-T1/CASP3* cells were exposed to HIV-1 and the cells were probed with Annexin V. At 6 h post-infection, 2.7\% of SUP-T1/ 
a

$b$
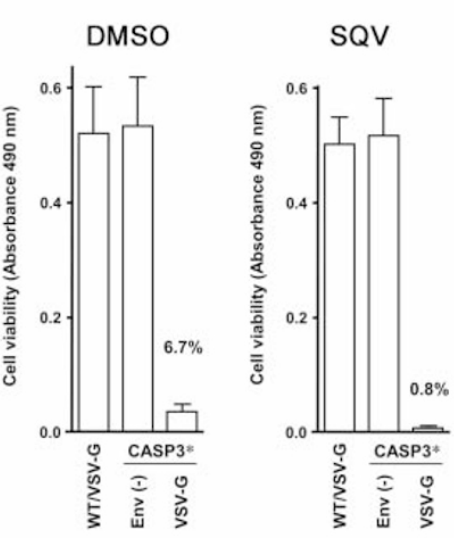

C
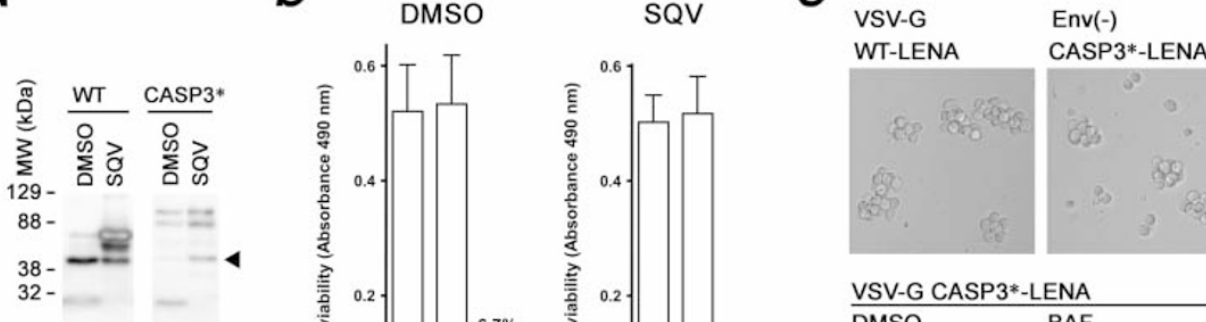

WT-LENA

CASP $3 *$-LENA

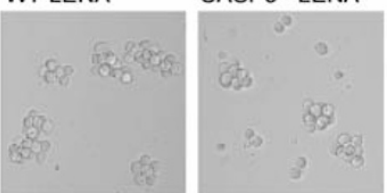

VSV-G CASP $3^{*}$-LENA

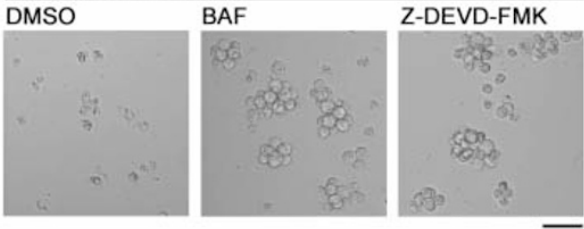

d

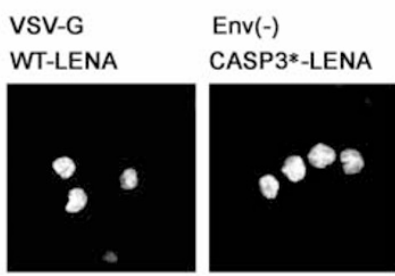

e

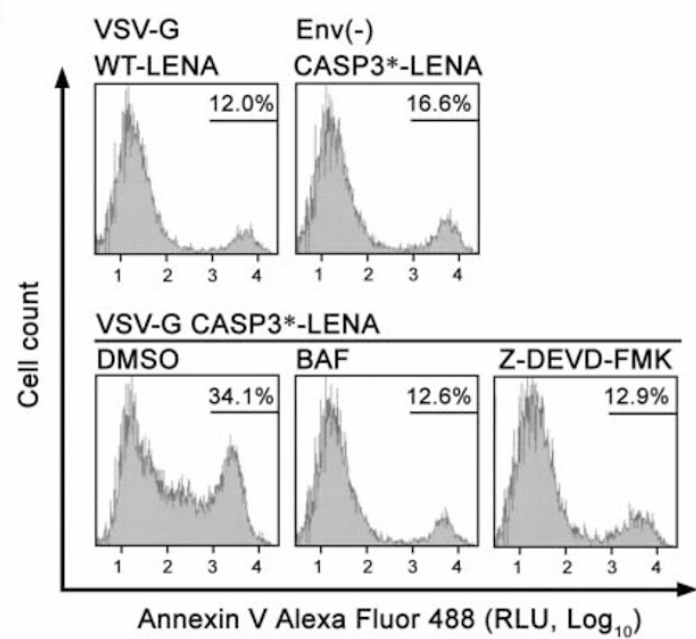

$f$

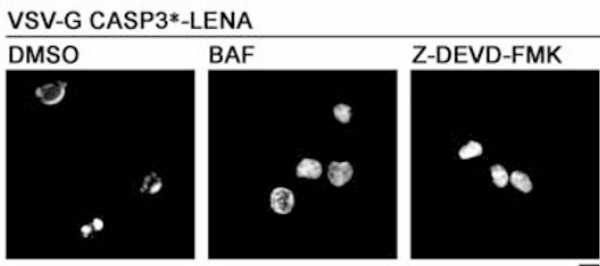

$g$

DMSO

BAF

Z-DEVD-FMK

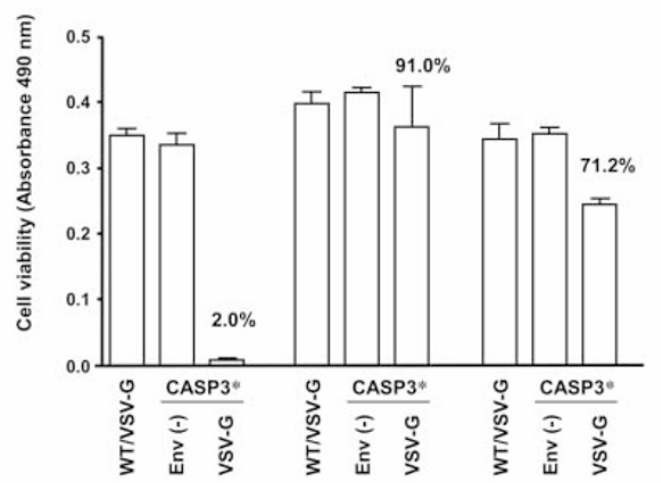

M8166

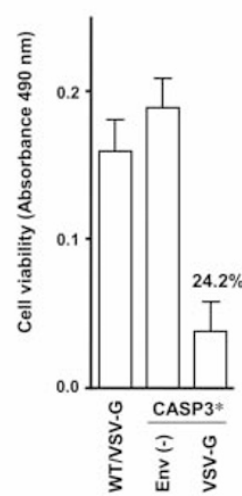

SUP-T1

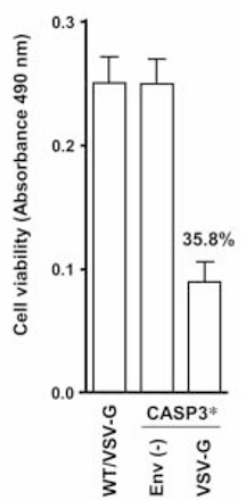

Figure $2 \mid$ Induction of apoptosis in leukemic cells by CASP3*-LENA. (a) Enhancement of WT- and CASP3*-LENA production by a low dose of saquinavir (SQV, $0.2 \mu \mathrm{M}$ ). DMSO was used as a solvent control. The arrowhead indicates a signal representing the MA-CA, indicating the release of CASP3* from CASP3*-Gag. (b) Quantitative analysis of MOLT-4 cell viability after LENA exposure. Cell viability was scored by colorimetric assay at 1d post-LENA exposure comparing WT-LENA encapsidated with VSV-G (WT/VSV-G), non-enveloped CASP3*-LENA (ENV(-)), and CASP3*-LENA encapsidated with VSV-G. The average and standard deviation from triplicated wells using LENA produced in the presence or absence of SQV are shown (SQV and DMSO, respectively). (c) Light microscopic observation of MOLT-4 cells treated with LENA produced in the presence of low-dose SQV. Cells were imaged at $1 \mathrm{~d}$ post-LENA exposure. Cells were treated with either Bafilomycin A1 (BAF) or the CASP3 inhibitor Z-DEVD-FMK. DMSO was used as a solvent control. Bar, $50 \mu \mathrm{m}$; magnification x100. (d) Nuclear morphology of MOLT-4 cells treated with LENA produced in the presence of low-dose SQV. Cells were imaged at $1 \mathrm{~d}$ post-LENA exposure after staining with Hoechst dye. Cells were treated with either BAF or Z-DEVD-FMK. DMSO was used as a solvent control. Bar, $10 \mu \mathrm{m}$; magnification x630. (e) Flow cytometric detection of apoptotic MOLT-4 cells. Cells were stained with Annexin V Alexa Fluor 488 at $6 \mathrm{~h}$ post-exposure to LENA produced in the presence of low dose nelfinavir (NFV, $0.2 \mu \mathrm{M})$. The live cell fraction was gated to analyze the early phase of apoptotic cell death. Cells were treated with either BAF or Z-DEVD-FMK. DMSO was used as a solvent control. RLU, relative light units. (f) Quantitative analysis of MOLT-4 cell viability after exposure to LENA produced in the presence of low-dose NFV $(0.2 \mu \mathrm{M})$. Cell viability was scored at $1 \mathrm{~d}$ post-LENA exposure. Cells were treated with either BAF or Z-DEVD-FMK. DMSO was used as a solvent control. (g) Quantitative analysis of M8166 and SUP-T1 cell viabilities as perfomed on MOLT-4 cells. 
a

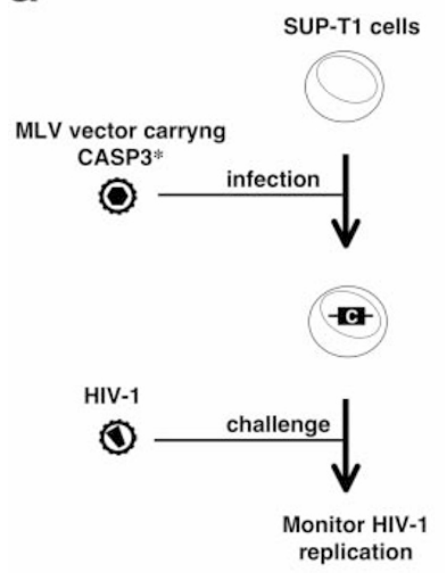

$b$

High-dose experiment

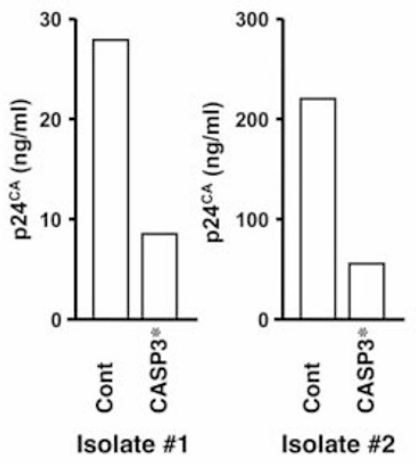

Low-dose experiment

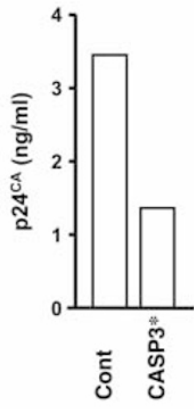

Isolate \#1

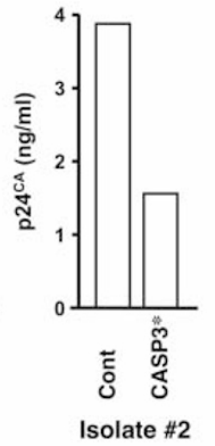

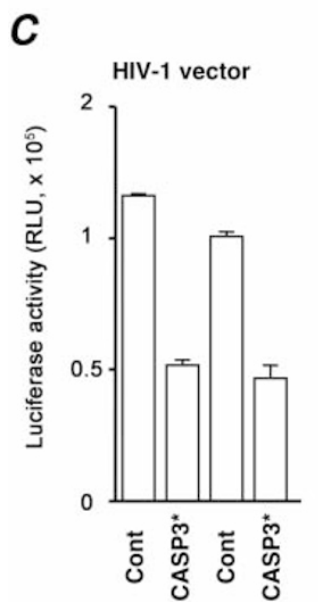

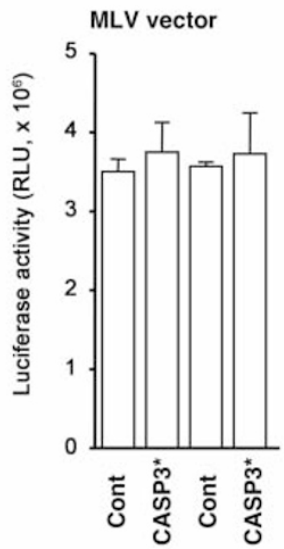

Expt \#1

Expt \#2
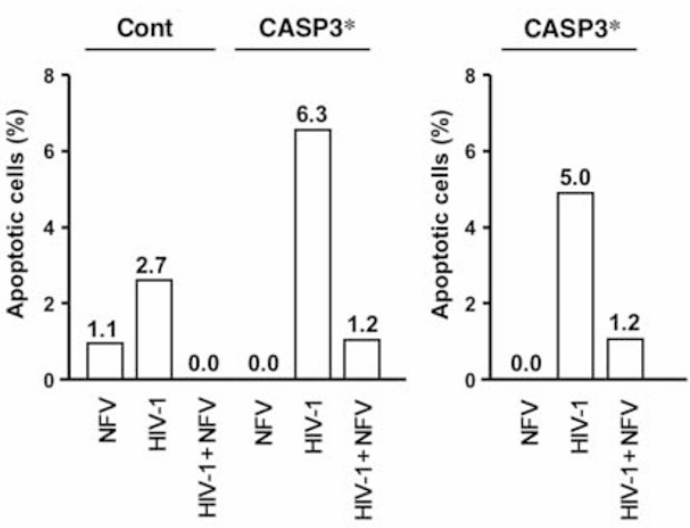

e
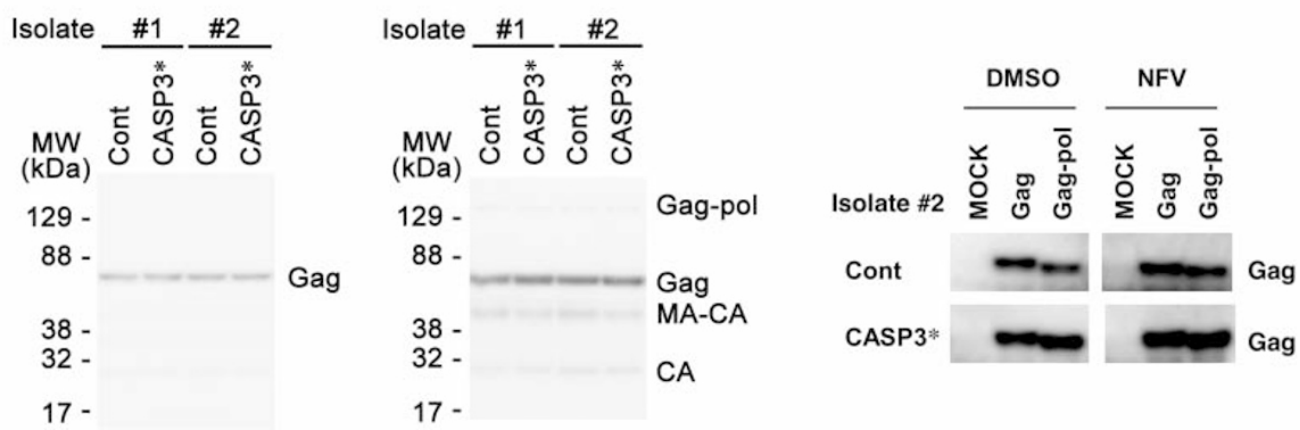

Figure 3 Inhibition of HIV-1 propagation by CASP3*. (a) Schematic representation of the experimental approach. SUP-T1 cells were infected with MLV carrying the CASP3* to produce cells constitutively expressing CASP $3^{*}$. These cells were infected with HIV-1, and the replication efficiency of HIV1 was measured using viral p $24^{\mathrm{CA}}$ antigen in the cell culture medium. (b) Inhibition of HIV-1 $1_{\mathrm{NL} 4-3}$ replication in SUP-T1/CASP $3 *$ cells. Cells were exposed to high- or low-dose viral preparation yielding $25 \mathrm{ng}$ or $1.3 \mathrm{ng}$ of $\mathrm{p} 24^{\mathrm{CA}}$ per $1 \times 10^{5}$ cells, respectively. The viral p2 $4^{\mathrm{CA}}$ concentration in the culture supernatant was measured at 6-9 d post-viral infection. Results shown were obtained from two independent isolates of SUP-T1/CASP3* and control cells. (c) Analysis of SUP-T1/CASP3* cell fate after HIV-1 entry. Luciferase activity of cells infected with either an HIV-1-based lentiviral vector or an MLV vector was measured at $4 \mathrm{~d}$ post-infection. RLU, relative light units. (d) Flow cytometric detection of apoptotic SUP-T1/Cont and SUP-T1/CASP3* cells by HIV-1 $1_{\mathrm{NL} 4-3}$ infection. The percentage of Annexin V Alexa Fluor 488 positive cells was measured at 6 h post-nelfinavir (NFV) exposure, post-HIV-1 infection, and post-HIV-1 infection in the presence of $2 \mu \mathrm{M} \mathrm{NFV}$, and subtracted from the percentage of Annexin V positive cells in untreated controls. The live cell fraction was gated according to scatter to analyze the early phase of apoptotic cell death. Data from two independent experiments are shown. For this experiment, cells from isolate \#2 were used. (e) Analysis of SUP-T1/CASP3* cell fate upon HIV-1 production. The SUP-T1/CASP3* cells were transduced with HIV-1 Gag-pol or Gag by either MLV vector (left panel) or transfection (right panel), and the expression of transduced proteins was detected at $7 \mathrm{~d}$ post-gene transduction by Western blot. For the transfection experiment, cells were maintained in the presence of $0.5 \mu \mathrm{M}$ NFV to detect the effect of Pol on cell survical. 
Cont cells underwent apoptosis, which was lowered by NFV (Fig. 3d), suggesting that the HIV-1 infection induces apoptotic cell death in a HIV-1 protease-dependent manner as previously reported $^{9,10}$. By contrast, the apoptotic cells was detected more frequently in SUP-T1/CASP3* cells than in SUP-T1/Cont cells $(6.3 \%$ and $5.0 \%$ in two independent experiments), which was drastically decreased to $1.2 \%$ in the presence of NFV (Fig. 3d). These data suggest that the CASP $3 *$ predispose cells to undergo apoptosis upon HIV-1 infection, and the virus-associated protease is responsible for the induction of apoptosis.

To examine the viral production phase, the HIV-1 gag-pol was transduced into SUP-T1/CASP3* cells by MLV vector, and the levels of Gag were evaluated. If $\mathrm{HIV}-1$ protease activity in virus producing cells is responsible for the activation of CASP $3 *$, then the expression of Gag-pol, not Gag, should confer the cell growth disadvantage, resulting in reduced levels of Gag in these cells. Transfected SUPT1/CASP3* cells were subjected to Western Blot analysis at $7 \mathrm{~d}$ postinfection. The levels of Gag and its proteolytic products in SUP-T1/ CASP3* cells were indistinguishable from those of the control cells (Fig. 3e). Similar results were obtained when SUP-T1/CASP3* cells were transfected with a plasmid expressing HIV-1 Gag-pol (Fig. 3e). These data indicate that the attenuation of HIV-1 replication in SUPT1/CASP $3 *$ cells is due to the activation of CASP $*$ by virion-associated HIV-1 protease at the viral entry phase, leading to the depletion of virus-infected cells from the culture by apoptosis. In agreement with this model, the inhibition of apoptosis by Z-DEVD-FMK enhanced the efficiency of HIV-1 replication (data not shown).

\section{Discussion}

This study provides evidence that CASP3* has therapeutic potential against cancer and HIV-1 infection, overcoming the potential difficulty of CASP3 for the application to human gene therapy ${ }^{3,411}$. The cancer therapy by CASP ${ }^{*}$-LENA may be more applicable to solid tumors due to the current technological limit in particle delivery. However, to apply the CASP3*-LENA approach to the cancer therapy, it is critical to improve the potency of CASP3*-LENA. This is because the cell killing activities of CASP3*-LENA in some transformed cells, including MT-4, a HTLV-1-transformed human T cell line, were undetectable although the LENA-mediated protein delivery by LENA has been shown to be highly efficient in MT- 4 cells ${ }^{6}$. MT-4 cells are highly resistant to apoptosis because they express potent anti-apoptotic genes, such as HTLV-1 $\operatorname{Tax}^{12-14}$. The Tax induces expression of intracellular caspase inhibitor, cFLIP, which inhibits the activation cycle of CASPs ${ }^{15,16}$. Cancer cells often express high levels of cellular inhibitor of apoptosis protein (CIAP), which may lower the susceptibility of cells to apoptosis induced by CASP3. We may be able to overcome this issue by generating a CASP3* mutant that does not interact with CIAP. The potentiation of CASP3*-LENA should also contribute to reduce the number of injections necessary to achieve the therapeutic effect. Additionally, for clinical applications, a regulatory system to control the cell tropism of LENA needs to be developed.

For HIV-1 infection, CASP3* may be applicable to adoptive stem cell therapy, alternatively termed intracellular vaccination. Stem cell gene therapy has been shown to be effective in clinical trials against HIV-1 infection, as has shRNA therapy targeting CCR5 mRNA ${ }^{7}$. Gene therapy using a zinc finger nuclease was found to be effective against HIV-1 infection as well ${ }^{8}$. These therapeutic approaches target nucleic acids, and off-target effects represent a serious concern. CASP ${ }^{*}$ is designed to be activated specifically by HIV-1 protease; thus, it offers a basis for a novel therapeutic approach with few "off-target" effects. CASP3* induces suicide in HIV-1-infected cells, and does not yield latently-infected cells. The CASP3* approach should be effective against drug-resistant HIV-1 strains since the anti-viral mechanism of CASP3* is distinct from those of currently-available anti-retroviral drugs. Furthermore, a CASP3*- resistant HIV-1 may not emerge easily. This is because mutations in viral protease that do not affect Gag processing but also do not activate CASP3* are unlikely to occur. Additionally, our strategy is applicable to other infectious diseases if the infectious agents encode proteases with unique substrate preferences. In summary, our work provides a platform for the development of a CASP $3 *$-based therapy against cancer and HIV-1 infection.

\section{Methods}

Plasmids. CASP3 was amplified from MT-4 total RNA using a two-step PCR protocol. For the first reaction, two RT-PCR reactions were performed using the following primer sets: fwd \# 15 '-acc ggt cAG CCA GAA CTA Ccc cat cgt gca gTC Tgg aat atc cct gga caa c-3' and rev \#1 5'-CTc acg atg ggG TAG TTC TGg tct gtc tca atg cca cag tc-3'; and fwd \#2 5'-acC AGA ACT ACc cca tcg tgA GTG GTG TTG ATG ATG ACA TGG-3' and rev \#2 5'-CAA TTG GTG ATA AAA ATA GAG TTC TTT TG-3'. The second PCR reaction was performed using fwd \#1 and rev \#2 primers targeting the products obtained in the first RT-PCR reactions. The AgeI-MfeI fragment of the PCR product was cloned into AgeI-EcoRI sites of plynMyGFP-gag-pol ${ }^{6}$, generating pCASP3*-gag-pol. The retroviral expression vector pQcCASP3* was constructed by amplifying CASP3* by PCR as detailed above using 5 '-acc ggt gcc acc ATG ggc TCT gga ata tcc ctg gac aac-3' and 5'-CAA TTG TTA GTG ATA AAA ATA GAG TTC TTT TG-3', and cloned into AgeI-EcoRI sites of pQcXIP (Clontech, Palo Alto, CA). The pCMMP Gagf vector was constructed by cloning a XhoI-BamHI fragment from pGag-GFP ${ }^{17}$ into the corresponding restriction sites of pCMMP KRAB ${ }^{18}$. The pCMMP HIV-1 gag-pol IRES GFP was constructed by three step cloning. First, the AgeI-BamHI KRAB gene fragment from pCMMP KRAB was cloned into the corresponding restriction sites of pCMMP LacZ IRES GFP $^{9}$, generating PCMMP KRAB IRES GFP. The AgeI-EcoRI BlaM gene fragment amplified from pUC19 by $\mathrm{PCR}^{6}$ was cloned into the AgeI-MfeI sites of pCMMP KRAB IRES GFP, generating pCMMP BlaM IRES GFP. The EcoRI-XhoI HIV-1 gag-pol gene fragment from pgag $\mathrm{pol}^{18}$ was cloned into the corresponding restriction sites of pCMMP BlaM IRES GFP, generating pCMMP HIV-1 gag-pol IRES GFP. The HIV-1 proviral DNA pNL4-3 was obtained from the NIH AIDS Research and Reference Program. The HIV-1 Gag expression vector pHIV-1 Gagf was produced by cloning an AgeI-BamHI fragment from pHIV-1 Gagf-GFP ${ }^{17}$ into the corresponding sites of pEGFP-C2 (Clontech).

Cells, viability assay, and transfection. Cells were maintained in RPMI 1640 medium (Sigma, St. Louis, MA) supplemented with 10\% fetal bovine serum (Japan Bioserum, Tokyo), $100 \mathrm{U} \mathrm{ml}^{-1}$ penicillin, and $100 \mu \mathrm{g} \mathrm{ml}^{-1}$ streptomycin (Invitrogen), at $37^{\circ} \mathrm{C}$ in a humidified $5 \% \mathrm{CO}_{2}$ atmosphere. Cells were transfected with Lipofectamine 2000 according to the manufacturer's protocol (Invitrogen). Bafilomycin A1 was used at a concentration of $12.5 \mathrm{ng} \mathrm{ml}^{-1}$ (Sigma), and a CASP3 inhibitor, Z-DEVD-FMK, was used at a concentration of $0.5 \mu \mathrm{M}$ (BioVision, Mountain View, CA). The HIV-1 protease inhibitors nelfinavir (NFV) and saquinavir (SQV) were obtained from the NIH AIDS Research and Reference Reagent Program. To produce VSV-G-pseudotyped LENA, $200 \mathrm{ng}$ pVSV-G (Clontech) and $2 \mu \mathrm{g}$ pCASP3*-gag-pol or pgag-pol ${ }^{18}$ were transfected into $293 \mathrm{~T}$ cells. Non-enveloped LENA was produced using pcDNA3 (Invitrogen) in place of pVSV-G. The LENA preparation was passed through nitrocellulose filters $(0.45 \mu \mathrm{m})$. Cell viability was scored by the CellTiter 96 Aqueous One Solution Cell Proliferation Assay (Promega). For the transfection of plasmids into SUP-T1 cells, a DEAE-dextran protocol was employed. Five million cells were resuspended in $250 \mu \mathrm{l}$ of STBS $(25 \mathrm{mM}$ TrisCl, pH 7.4, $137 \mathrm{mM} \mathrm{NaCl}, 5 \mathrm{mM} \mathrm{KCl}, 0.6 \mathrm{mM} \mathrm{Na}_{2} \mathrm{HPO}_{4}, 0.7 \mathrm{mM} \mathrm{CaCl}_{2}$, and $0.5 \mathrm{mM}$ $\mathrm{MgCl}_{2}$ ), including $10 \mu \mathrm{g}$ of plasmid DNA. Cells were mixed with $250 \mu \mathrm{l}$ of STBS containing $1 \mathrm{mg} \mathrm{ml}^{-1}$ of DEAE-dextran and incubated for $30 \mathrm{~min}$ at room temperature (RT). The cells were then incubated with STBS containing 10\% DMSO for $2 \mathrm{~min}$ at RT and washed with $1 \mathrm{ml} \mathrm{HBSS}$ (Invitrogen)

Protein transduction. Protein transduction was performed as described previously by incubating approximately $1 \times 10^{6}$ cells with $1 \mathrm{ml}$ LENA-containing culture medium at $37^{\circ} \mathrm{C}^{3}$

Immunological detection. The detection of viral gene products by Western blot analysis was performed as described previously ${ }^{19}$, except that the anti-p24 ${ }^{\mathrm{CA}}$ monoclonal antibody clone $183-\mathrm{H} 12-5 \mathrm{C}$ and anti-CASP3 p17 subunit polyclonal antibody (Cat. \# AB3623, Millipore, Tokyo, Japan) were used. Immunofluorescent analysis was performed as described previously using the same monoclonal antibody ${ }^{18}$. Signals were detected using an LAS-3000 mini Lumino-Image analyzer operated by the LAS-300mini Image Reader software (ver.2.2, Fuji Film, Tokyo, Japan). The brightness and contrast of the image were adjusted using Adobe Photoshop (ver.7.0, Adobe, Tokyo, Japan).

Microscopy. Cells were imaged by confocal fluorescence microscopy (LSM510 Meta, Carl Zeiss MicroImaging Inc., Tokyo, Japan). For the imaging of nuclei, multiple focal planes were projected to generate a single image.

Virus production and infection. The HIV-1, lentiviral and MLV vectors used have been described previously'. The pQcXIP was used as a control for pQcCASP3*. 
Detection of CASP3 enzymatic activity. The substrate for the CASP3 enzyme assay was purchased from Promega (Caspase-Glo 3/7 Assay System, Promega, Madison, WI). Luciferase activity was measured using the Steady-Glo Luciferase Assay system (Promega). Luminescence was detected using a Veritas ${ }^{\mathrm{TM}}$ Microplate Luminometer (Promega).

Annexin V apoptosis assay. Cells were analyzed by flow cytometry using the Vybrant Apoptosis Assay kit (Invitrogen).

Enzyme-Linked Immunosorbent Assay (ELISA). A p24 ELISA was conducted according to the manufacturer's protocol (Zeptometrics, Buffalo, NY). To measure cellular p24, transfected 293T cells were washed once with PBS, lysed in $500 \mu \mathrm{l}$ buffer A (described above) for $30 \mathrm{~min}$, and then subjected to the ELISA.

1. Cohen, G. M. Caspases: the executioners of apoptosis. Biochem J. 326, 1-16 (1997).

2. Boatright, K. M. \& Salvesen, G. S. Mechanisms of caspase activation. Curr Opin Cell Biol. 15, 725-31 (2003).

3. Yamabe, K. et al. Cancer gene therapy using a pro-apoptotic gene, caspase-3. Gene Ther. 6, 1952-9 (1999).

4. Cam, L., Boucquey, A., Coulomb-L'hermine, A., Weber, A. \& Horellou, P. Gene transfer of constitutively active caspase- 3 induces apoptosis in a human hepatoma cell line. J Gene Med. 7, 30-8 (2005).

5. Walters, J. et al. A constitutively active and uninhibitable caspase- 3 zymogen efficiently induces apoptosis. Biochem J. 424, 335-45 (2009).

6. Aoki, T., Miyauchi, K., Urano, E., Ichikawa, R. \& Komano, J. Protein transduction by pseudotyped lentivirus-like nanoparticles. Gene Ther 18, 936-941 (2011).

7. Mitsuyasu, R. T. et al. Phase 2 gene therapy trial of an anti-HIV ribozyme in autologous CD34+ cells. Nat Med. 15, 285-92. Epub 2009 Feb 15. (2009).

8. Holt, N. et al. Human hematopoietic stem/progenitor cells modified by zincfinger nucleases targeted to CCR5 control HIV-1 in vivo. Nat 28, 839-47 (2010).

9. Komano, J., Miyauchi, K., Matsuda, Z. \& Yamamoto, N. Inhibiting the Arp2/3 complex limits infection of both intracellular mature vaccinia virus and primate lentiviruses. Mol Biol Cell. 15, 5197-207. Epub 2004 Sep 22. (2004).

10. Nicholson, D. W. et al. Identification and inhibition of the ICE/CED-3 protease necessary for mammalian apoptosis. Nature. 376, 37-43 (1995).

11. Wagner, R. et al. Rev-independent expression of synthetic gag-pol genes of human immunodeficiency virus type 1 and simian immunodeficiency virus: implications for the safety of lentiviral vectors. Hum Gene Ther 11, 2403-13 (2000)

12. Shaulian, E. \& Karin, M. AP-1 as a regulator of cell life and death. Nat Cell Biol. 4 E131-6 (2002).

13. Azran, I., Schavinsky-Khrapunsky, Y. \& Aboud, M. Role of Tax protein in human T-cell leukemia virus type-I leukemogenicity. Retrovirology. 1, 20 (2004).
14. Charoenthongtrakul, S., Zhou, Q., Shembade, N., Harhaj, N. S. \& Harhaj, E. W. Human T cell leukemia virus type 1 Tax inhibits innate antiviral signaling via NFkappaB-dependent induction of SOCS1. J Virol. 85, 6955-62. Epub 2011 May 18. (2011).

15. Krueger, A. et al. HTLV-1 Tax protects against CD95-mediated apoptosis by induction of the cellular FLICE-inhibitory protein (c-FLIP). Blood. 107, 3933-9. Epub 2006 Jan 10 (2006).

16. Okamoto, K., Fujisawa, J., Reth, M. \& Yonehara, S. Human T-cell leukemia virus type-I oncoprotein Tax inhibits Fas-mediated apoptosis by inducing cellular FLIP through activation of NF-kappaB. Genes Cells. 11, 177-91 (2006).

17. Aoki, T. et al. Improvement of lentiviral vector-mediated gene transduction by genetic engineering of the structural protein Pr55 Gag. Gene Ther 17, 1124-33 (2010).

18. Urano, E. et al. Substitution of the myristoylation signal of human immunodeficiency virus type 1 Pr55Gag with the phospholipase C-delta pleckstrin homology domain results in infectious pseudovirion production. J Gen Virol. 89, 3144-9 (2008).

19. Shimizu, S. et al. Inhibiting lentiviral replication by HEXIM1, a cellular negative regulator of the CDK9/cyclin T complex. Aids. 21, 575-82 (2007).

\section{Acknowledgments}

This work was supported by the Japan Health Science Foundation, the Japanese Ministry of Health, Labor, and Welfare (H18-AIDS-W-003 to JK), and the Japanese Ministry of Education, Culture, Sports, Science and Technology (18689014 and 18659136 to JK).

\section{Author contributions}

K.M., E.U., M.T., R.I., and J.K. designed and performed experiments and interpret the data J.K. wrote the manuscript. K.M. and E.U. equally contributed to this work.

\section{Additional information}

Competing financial interests: The authors declare no competing financial interests.

License: This work is licensed under a Creative Commons

Attribution-NonCommercial-NoDerivative Works 3.0 Unported License. To view a copy of this license, visit http://creativecommons.org/licenses/by-nc-nd/3.0/

How to cite this article: Miyauchi, K., Urano, E., Takizawa, M., Ichikawa, R. \& Komano, J. Therapeutic potential of HIV protease-activable CASP3. Sci. Rep. 2, 359; DOI:10.1038/ srep00359 (2012) 